\title{
Assessment and treatment of the failing right heart: considerations for transplantation referral
}

\author{
Ziad Taimeh \\ Texas Heart Institute, Baylor College of Medicine, Houston, TX, USA \\ Correspondence to: Ziad Taimeh, MD. Advanced Heart Failure, Mechanical Circulatory Support, and Heart Transplantation. Texas Heart Institute, \\ Baylor College of Medicine, Houston, TX, USA. Email: Ziad.Taimeh@bcm.edu.
}

\begin{abstract}
Long considered an insignificant chamber, the right ventricle has been recently under spotlight, especially with emerging data supporting its critical role in disease progression. The right ventricle is a triangular heart chamber with complex physiology and pathophysiology. In this review, we discuss the normal physiology of the right ventricle, right ventricular failure, and recent advances in treatment with emphasis on optimal timing for transplantation referral.
\end{abstract}

Keywords: Right ventricle; right ventricular failure; pulmonary hypertension; heart failure; mechanical circulatory support; heart transplantation; lung transplantation

Submitted Jun 03, 2019. Accepted for publication Aug 12, 2019.

doi: $10.21037 /$ jtd.2019.08.60

View this article at: http://dx.doi.org/10.21037/jtd.2019.08.60

\section{Introduction}

Starr et al. (1) and Bakos (2) demonstrated in the 1940s that induced right ventricular (RV) myocardial necrosis in canines did not result in significant consequences on animal performance. Animals with surgically removed RV free wall could live a normal sedentary life (3). Thus, the RV has long been considered an accessory chamber needed only in cases of high RV afterload. This is until recent years when the true significance of RV function has been under spotlight, especially after advances in knowledge of the pathophysiology of pulmonary arterial hypertension (4).

\section{Anatomy and physiology of the right ventricle}

The RV is complex crescentic in shape with an inflow (sinus), trabeculated body, and outflow (infundibulum). The inflow valve is tricuspid in structure and connected via chordae tendineae to the musculi papillares. The outflow valve is semi-lunar (pulmonary valve). The RV has coarser trabeculations and a thinner wall than the left ventricle (LV) (5). It contracts in a peristaltic fashion from the inflow to the outflow utilizing longitudinal fiber shortening and is primarily dependent on the interventricular septum to propel blood to the pulmonary circulation (5).

The RV is a low-pressure, high-volume chamber with a trapezoid flow-volume loop. It is extremely sensitive to its loading conditions (6). The normal RV fills at or below its unstressed volume, such that changes in RV end-diastolic volume occur without any change in RV diastolic wall stretch. The RV afterload is dependent on the arterial elastance of the pulmonary circulation, constituting the basis for RV-arterial coupling (7). Once the RV afterload increases in conditions such as pulmonary arterial hypertension, RV contractility becomes essential to maintain the cardiac output.

\section{RV failure}

Due to its complex nature, several definitions exist to describe RV failure. According to the International Right Heart Foundation Working Group, RV failure is broadly defined as a clinical syndrome due to an alteration of structure and/or function of the right heart circulatory system, leading to deranged forward flow to the pulmonary circulation. The right heart circulatory system is made of the systemic veins, right atrium, RV proper, and the pulmonary arteries up to the capillaries (8). 
RV dysfunction can be caused by contractility or pump failure, volume overload (increased preload), or pressure overload (increased afterload). The RV contractility reserve can be compromised by myocardial ischemia or infarction (9), myocarditis (10), or congenital diseases such as arrhythmogenic RV dysplasia (11). On the other hand, volume overload could be caused by primary tricuspid valve regurgitation. However, $\mathrm{RV}$ failure is primarily attributed to pressure overload in cases such as left heart failure, pulmonary arterial hypertension, and pulmonary embolism (12). The RV is especially dependent on afterload, and even slight changes in pulmonary vascular resistance can reduce the RV cardiac output (5).

\section{Assessment of the right ventricle}

No single sign, symptom, or laboratory test can accurately identify RV failure. Nonetheless, certain clues are suggestive of $\mathrm{RV}$, rather than $\mathrm{LV}$, failure. For instance, abdominal distention and ascites signify RV failure rather than $\mathrm{LV}$ failure, which presents as pitting edema in dependent body parts. Syncope, shortness of breath, and fatigue are also common clinical signs of advanced RV failure (13).

The intricate triangular shape of the RV (and crescentic in cross-section) requires multimodal imaging and hemodynamic approaches for accurate assessment. Echocardiography is widely used in current practice to assess the gross structure and systolic function of the RV. For instance, RV chamber size at the base $>4.2 \mathrm{~cm}$, a fractional change of $<35 \%$, a tricuspid annular plane systolic excursion (TAPSE) $<1.6 \mathrm{~cm}$, and tissue Doppler S' $<10 \mathrm{~cm} / \mathrm{sec}$ constitute a dilated RV with depressed systolic function (13). More recent cardiac magnetic resonance imaging-derived $\mathrm{RV}$ parameters, such as RV mass and RV volumes, are evolving as gold-standard for RV structural assessment and continue to show promise with increasing significance (14). Finally, invasive hemodynamic evaluation of the RVpulmonary circulation remains essential for advanced treatment. Right side cardiac filling pressures, namely right atrial pressure, pulmonary artery pressure, cardiac output, pulmonary vascular resistance, and RV stroke work have been previously associated with outcome data in patients with RV failure (15).

\section{Treatment of RV failure}

Early recognition of a dysfunctional RV, before progression to RV failure, is critical for optimal outcome. Expert opinions vary on what constitutes "RV dysfunction" $v$.
"RV failure". It is plausible to describe RV dysfunction as abnormality in RV imaging (size or systolic function). Whereas RV failure is the clinical syndrome of right sided heart failure, described as markedly elevated right sided cardiac filling pressures and low cardiac output. Therapy for RV dysfunction/failure should be directed at both the underlying etiology, as well as the failing right heart circulatory system. Treatment of the underlying etiology varies based on the pathophysiology involved and is out of the scope of this review. For instance, treatment of pulmonary arterial hypertension focuses on pulmonary vasodilators.

Once etiology-specific treatment of RV failure is implemented, support of right heart circulatory system should include optimization of right-side filling pressures and cardiac output. This includes fluid management with diuretics or renal replacement therapy, inotropic support for hypoperfusion, and mechanical circulatory support if needed (16). The goal of fluid management is to ensure adequate preload for cardiac filling, while providing relief from RV volume overload maintaining the triangular geometry of the RV needed for optimal contractility. Previous studies suggest that almost $70 \%$ of cases of RV failure could be compensated by regaining the optimal geometry of the RV (17). When optimizing volume status is unsuccessful, initiation of inotropic support becomes needed to maintain body perfusion. No single inotrope has shown superiority in cases of RV failure; however, milrinone seems better suited for right heart circulatory failure. It improves RV contractility and lowers RV afterload simultaneously. One caveat is maintaining RV coronary perfusion. Because tissue pressure in the $\mathrm{LV}$ rises during systole to systemic levels, coronary perfusion of the $\mathrm{LV}$ is primarily during diastole. However, pressure in the RV does not normally exceed systemic systolic pressure, thus coronary flow to the RV happens during diastole and systole. That being said, maintaining systemic systolic blood pressure at least $10-15 \mathrm{mmHg}$ above pulmonary artery systolic pressure will ensure adequate RV perfusion (18).

In recent years, mechanical circulatory support has emerged as means to maintain perfusion and/or oxygenation. Traditional Extracorporeal membrane oxygenation (ECMO) can be implemented in different orientations. Venoarterial ECMO bypasses both the heart and lungs, providing perfusion and oxygenation support. Venovenous ECMO bypasses the lungs only, providing oxygenation support only (19). Other temporary mechanical circulatory support devices include the Impella $\mathrm{RP}^{\circledR}$ (Abiomed, Aachen, Germany), 
TANDEM Protek DUO ${ }^{\circledR}$ (CardiacAssist, Pittsburgh, PA) with or without an oxygenator, and Centrimag ${ }^{\circledR}$ (Lake Bluff, Illinois) (20). The Impella $\mathrm{RP}^{\circledast}$ did show improved outcomes in the RECOVER RP Clinical Trial. It is a micro-axial flow pump inserted via the femoral vein (21). It only supports perfusion without the ability to improve oxygenation. The TANDEM ${ }^{\circledR}$ platform is a centrifugal pump that can be adapted for RV support with the option of oxygenator. Finally, the Centrimag pump is a surgically inserted pump typically used in bridging patients for heart transplantation.

In cases of irreversible RV failure, options are more limited. Durable RV assist devices (HeartWare, Framingham, MA) or total artificial heart implantation have been previously attempted with promising success (22). Yet, heart, lung, or combined heart-lung transplantation is the gold standard for ultimate treatment (23). The transplantation strategy depends on several factors, primarily driven by the inciting etiology of RV failure. In cases of RV failure due to left heart disease, heart transplantation is appropriate. In cases of $\mathrm{RV}$ failure due to pulmonary artery hypertension, the decision to proceed with lung $v s$. combined heart-lung transplantation is complex and controversial. In general, If the $\mathrm{LV}$ ejection fraction (EF) is $>50 \%$, RVEF is $>25 \%$, and RV scarring is absent on cardiac MRI, then isolated lung transplantation is more appropriate. However, in the presence of Eisenmenger physiology, LVEF $<30 \%$, RVEF $<10 \%$, and RV scarring is extensive on cardiac MRI, then combined heart-lung transplantation is needed (24). Unfortunately, heart-lung transplantation is associated with the worse post-transplant outcomes. According to the International Society of Heart and Lung Transplantation Adult Registry, 3,998 combined heart-lung transplants occurred from 1982 to 2016, with median survival of 3.4 years (23), which is lower than heart-only (median survival of 10.7 years) or lung-only (median survival of 6.2 years) transplantation $(23,25)$. Thus, lung transplantation should be considered early in the disease course to avoid progression to RV failure.

Once all more viable options are exhausted, palliative procedures such as balloon atrial septostomy or Potts shunting could be considered. In atrial septostomy, an inter-atrial septal defect is created to vent the high filling pressure from the right to the left atrium, attempting to improve cardiac output. Unfortunately, the resulting hypoxia limits the utility of this strategy (26). The Potts shunting is another technique for decompressing the RV without significant upper body cyanosis. This is a surgical procedure involving side-to-side anastomosis connecting the left pulmonary artery to the descending aorta, resulting in a right-to-left shunt (27). Both techniques are primarily used for palliative purposes, and in some cases as bridge to transplantation.

\section{Conclusions}

The $R V$ is a very complex chamber with a unique physiology. It is becoming clear that involvement of the $\mathrm{RV}$ in disease pathophysiology significantly worsens outcomes. Early referral for advanced treatment such as transplantation should be considered, as appropriate.

\section{Acknowledgments}

None.

\section{Footnote}

Conflicts of Interest: The author has no conflicts of interest to declare.

Ethical Statement: The author is accountable for all aspects of the work in ensuring that questions related to the accuracy or integrity of any part of the work are appropriately investigated and resolved.

\section{References}

1. Starr I, Jeffers W, Mead R. The absence of Conspicuous increments of venous pressure after severe damage to the right ventricle of the $\mathrm{dog}$, with a discussion of the relation between clinical congestive failure and heart disease. Am Heart J 1943;26:291.

2. Bakos ACP. The question of the function of the right ventricular myocardium: an experimental study. Circulation 1950;1:724-32.

3. Donald DE, Essex HE. Pressure studies after inactivation of the major portion of the canine right ventricle. Am J Physiol 1954;176:155-61.

4. Grinstein J, Gomberg-Maitland M. Management of pulmonary hypertension and right heart failure in the intensive care unit. Curr Hypertens Rep 2015;17:32.

5. Sheehan F, Redington A. The right ventricle: anatomy, physiology and clinical imaging. Heart 2008;94:1510-5.

6. Friedberg MK, Redington AN. Right versus left ventricular failure: differences, similarities, and 
interactions. Circulation 2014;129:1033-44.

7. Muntean I, Benedek T, Togănel R. Right ventriculoarterial coupling in heart failure - Is the right ventricle independent? Int J Cardiol 2018;259:136.

8. Mehra MR, Park MH, Landzberg MJ, et al. Right heart failure: toward a common language. J Heart Lung Transplant. 2014;33:123-6.

9. Namana V, Gupta S, Abbasi A, et al. Right ventricular infarction. Cardiovasc Revasc Med 2018;19:43-50.

10. Aquaro GD, Negri F, De Luca A, et al. Role of right ventricular involvement in acute myocarditis, assessed by cardiac magnetic resonance. Int J Cardiol. 2018;271:359-65.

11. Haugaa KH, Haland TF, Leren IS, et al. Arrhythmogenic right ventricular cardiomyopathy, clinical manifestations, and diagnosis. Europace 2016;18:965-72.

12. Greyson CR. Pathophysiology of right ventricular failure. Crit Care Med 2008;36:S57-65.

13. Konstam MA, Kiernan MS, Bernstein D, et al. Evaluation and Management of Right-Sided Heart Failure: A Scientific Statement From the American Heart Association. Circulation 2018;137:e578-622.

14. Surkova E, Muraru D, Iliceto S, et al. The use of multimodality cardiovascular imaging to assess right ventricular size and function. Int J Cardiol 2016;214:54-69.

15. Howard LS. Prognostic factors in pulmonary arterial hypertension: assessing the course of the disease. Eur Respir Rev 2011;20:236-42.

16. Harjola VP, Mebazaa A, Čelutkienė J, et al. Contemporary management of acute right ventricular failure: a statement from the Heart Failure Association and the Working Group on Pulmonary Circulation and Right Ventricular Function of the European Society of Cardiology. Eur J Heart Fail 2016;18:226-41.

17. Grignola JC, Domingo E. Acute Right Ventricular Dysfunction in Intensive Care Unit. Biomed Res Int 2017;2017:8217105.

18. Lowensohn HS, Khouri EM, Gregg DE, et al. Phasic right coronary artery blood flow in conscious dogs with

Cite this article as: Taimeh Z. Assessment and treatment of the failing right heart: considerations for transplantation referral. J Thorac Dis 2019;11(Suppl 14):S1817-S1820. doi: 10.21037/ jtd.2019.08.60 normal and elevated right ventricular pressures. Circ Res 1976;39:760-6.

19. Simon MA. Assessment and treatment of right ventricular failure. Nat Rev Cardiol 2013;10:204-18.

20. Sultan I, Kilic A, Kilic A. Short-Term Circulatory and Right Ventricle Support in Cardiogenic Shock: Extracorporeal Membrane Oxygenation, Tandem Heart, CentriMag, and Impella. Heart Fail Clin 2018;14:579-83.

21. Anderson MB, Goldstein J, Milano C, et al. Benefits of a novel percutaneous ventricular assist device for right heart failure: The prospective RECOVER RIGHT study of the Impella RP device. J Heart Lung Transplant 2015;34:1549-60.

22. Bernhardt AM, De By TM, Reichenspurner H, et al. Isolated permanent right ventricular assist device implantation with the HeartWare continuous-flow ventricular assist device: first results from the European Registry for Patients with Mechanical Circulatory Support. Eur J Cardiothorac Surg 2015;48:158-62.

23. Chambers DC, Yusen RD, Cherikh WS, et al. The Registry of the International Society for Heart and Lung Transplantation: Thirty-fourth Adult Lung And HeartLung Transplantation Report-2017; Focus Theme: Allograft ischemic time. J Heart Lung Transplant 2017;36:1047-59.

24. Olland A, Falcoz PE, Canuet M, et al. Should we perform bilateral-lung or heart-lung transplantation for patients with pulmonary hypertension? Interact Cardiovasc Thorac Surg 2013;17:166-70.

25. Lund LH, Khush KK, Cherikh WS, et al. The Registry of the International Society for Heart and Lung Transplantation: Thirty-fourth Adult Heart Transplantation Report-2017; Focus Theme: Allograft ischemic time. J Heart Lung Transplant 2017;36:1037-46.

26. Norfolk SG, Lederer DJ, Tapson VF. Lung transplantation and atrial septostomy in pulmonary arterial hypertension. Clin Chest Med 2013;34:857-65.

27. Kim SH, Jang WS, Lim HG, Kim YJ. Potts shunt in patients with primary pulmonary hypertension. Korean J Thorac Cardiovasc Surg 2015;48:52-4. 